\title{
АНАЛИЗ ДИНАМИЧЕСКИХ ПРОЦЕССОВ СИСТЕМЫ АВТОМАТИЧЕСКОГО РЕГУЛИРОВАНИЯ НАПРЯЖЕНИЯ ПРЕОБРАЗОВАТЕЛЯ С ШИМ
}

\author{
Асп. Е.Я. Ивакина
}

\section{АНАЛІЗ ДИНАМІЧНИХ ПРОЦЕСІВ СИСТЕМИ АВТОМАТИЧНОГО РЕГУЛЮВАННЯ НАПРУГИ ПЕРЕТВОРЮВАЧА 3 ШІМ}

\author{
Асп. К.Я. Івакіна
}

\section{THE ANALYSIS OF DYNAMIC PROCESSES OF THE SYSTEM OF AUTOMATIC IS ADJUSTING OF TENSION OF TRANSFORMER WITH SHIM}

\author{
Postgraduate K.I. Ivakina
}

У статті виконано аналіз динамічних прочесів системи автоматичного регулювання, яка має випрямляч з широтно-імпульсною модулячією. Показано вирівнюючу дію фактора пульсачій на зміни коефіцієнта передачі перетворювача.

Ключові слова: випрямляч, широтно-імпульсна модулячія, замкнута структура, коефіцієнт передачі, фактор пульсацій, процес кінщевої тривалості.

В статье выполнен анализ динамических прочессов системы автоматического регулирования, содержащей выпрямитель с ииротно-импульсной модуляиией выходного напряжения. Показано выравнивающее действие фактора пульсаций на изменения коэффициента передачи выпрямителя.

Ключевые слова: выпрямитель, широтно-импульсная модуляция, замкнутая структура, коэффищиент передачи, фактор пульсаций, прочесс конечной длительности.

In the article the analysis of dynamic processes of the system of automatic control, containing a rectifier with latitudinal-impulsive modulation of output tension, is executed. The analysis of dynamic processes is executed for the system of automatic control of output tension of rectifier with an integral regulator. The analysis of dynamic processes is executed with the use of impulsive model of transformer and mathematical vehicle $Z$ - transformations. The condition of process of eventual duration is got. It is shown, that due to the changes of factor of pulsations influence on the transitional process of changes of coefficient of transmission of rectifier is compensated. The results of theoretical researches are confirmed by the results of imitation design in the Matlab environment.

Keywords: rectifier, latitudinal-impulsive modulation, reserved structure, coefficient of transmission, factor of pulsations, process of eventual duration.

Введение. Создание мощных стабилизированных систем питания для железнодорожного транспорта напрямую связано с динамическими характеристиками полупроводникового преобразователя электрической энергии.

Постановка проблемы в общем виде и ее связь с важными научными и практическими заданиями. Повышение качества электрической энергии в входной и выходной цепях полупроводниковых преобразователей является актуальной задачей. Решение этой задачи напрямую связано с динамическими характеристиками преобразователя как элемента замкнутой структуры автоматического регулирования. 
Анализ последних достижений и публикаций. Динамические характеристики тиристорного выпрямителя и широтно-импульсного преобразователя в разных режимах работы достаточно изучены и изложены в ряде работ, среди которых следует отметить $[1,2,3,4,5]$.

В работах $[1,2,4]$ получены импульсные модели тиристорного выпрямителя для режимов непрерывного и прерывистого токов, с учетом и без учета угла коммутации.

В работах $[3,4,5]$ изложены импульсные модели широтно-импульсного преобразователя для разных режимов в цепи нагрузки при постоянстве величины входного напряжения.

Анализ результатов исследований, приведенных в перечисленных работах, указывает на то, что повышение динамических характеристик напрямую связано с уменьшением длительности тактового интервала преобразователя, что эквивалентно расширению его полосы пропускания.

Одним из путей улучшения частотных свойств выпрямителя является применение в нем вместо фазового управления широтно-импульсной модуляции (ШИМ) [5]. Объединение в преобразователе свойств выпрямителя и широтно-импульсного преобразователя вносит коррективы в протекании динамических процессов.

Определение цели и задачи исследований. Цель работы заключается в теоретических и экспериментальных исследованиях динамических процессов в замкнутых структурах, содержащих выпрямитель с ШИМ.

Основная часть исследований. Выпрямитель с ШИМ при малых отклонениях управляющего воздействия $\Delta U_{y}(t)$ представляет собой амплитудноимпульсный модулятор второго рода. Импульсная модель выпрямителя с ШИМ для режима непрерывного тока изображена на рис. 1.

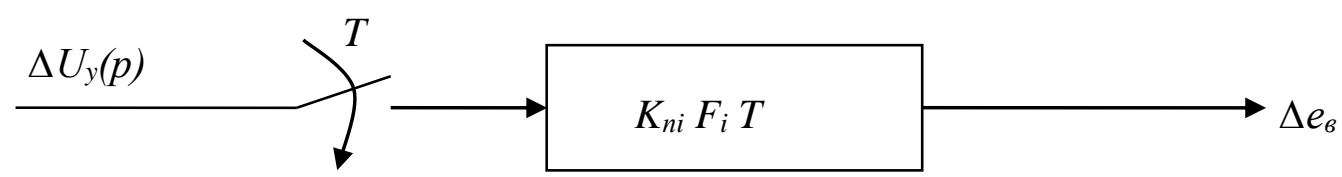

Рис. 1. Импульсная модель выпрямителя с ШИМ

Особенностью импульсной модели является то, что статический коэффициент передачи $K_{n i}$ и фактор пульсаций $F_{i}$ являются переменными величинами. Вызвано это воздействием пульсационной составляющей выходного напряжения выпрямителя на импульсную последовательность ШИМ. В силу того, что коэффициент передачи $K_{n i}$ и фактор пульсаций $F_{i}$ связаны между собой, следует ожидать ослабление влияния их изменений на динамические свойства замкнутой системы автоматического регулирования. Для исследования данной предпосылки рассмотрим замкнутую систему автоматического регулирования выходного напряжения выпрямителя с ШИМ с интегральным регулятором, передаточная функция которого

$$
\mathrm{G}(\mathrm{p})=\frac{1}{\mathrm{~T}_{1} p}
$$

Выбор такой структуры объясняется возможностью реализации предельного быстродействия. Структурная схема рассматриваемой системы изображена на рис. 2. 


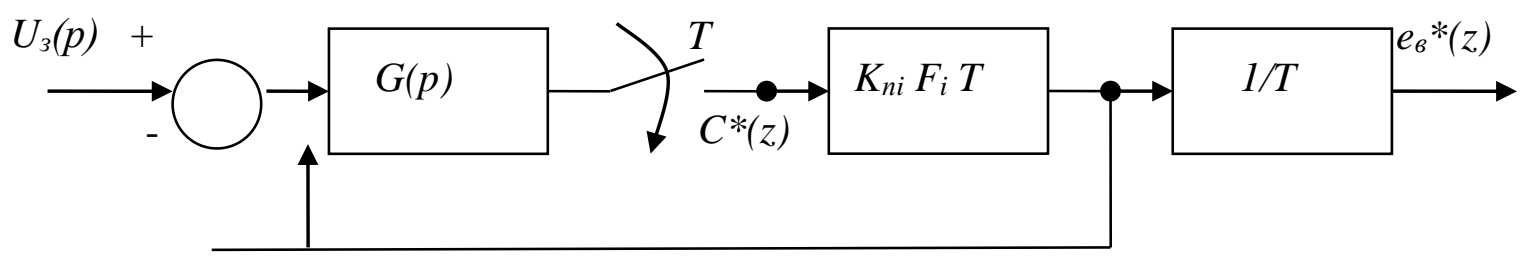

Рис. 2. Структурная схема системы регулирования

Связь между входным $U_{3}(p)$ и выходным $e_{6} *(z)$ сигналами, записанная в форме z- преобразования, определяется следующим образом:

$$
\begin{aligned}
& e_{B}^{*}(\mathrm{z})=\mathrm{C} *(\mathrm{z}) \cdot \mathrm{K}_{\text {пі }} F_{i} T \text {; } \\
& C *(z)=\frac{U_{3} \mathrm{G}^{*}(\mathrm{z})}{1+\mathrm{K}_{\mathrm{ni}} F_{i} T G^{*}(z)} ; \\
& e_{\mathrm{B}}^{*}(\mathrm{z})=\frac{\mathrm{K}_{\mathrm{ni}} F_{i} T \cdot U_{3} \mathrm{G} *(\mathrm{z})}{1+\mathrm{K}_{\mathrm{ni}} F_{i} T G *(z)} ;
\end{aligned}
$$

В силу того, что разность порядков полиномов знаменателя и числителя передаточной функции (1) приведенной непрерывной части системы равна единице, еe реакция на последовательность $\delta(t)$ импульсов, формируемых импульсным элементом, равна единице. Для фиксации импульсным элементом левосторонних значений выходного сигнала регулятора $G(p)$ представим выражение (2) в следующем виде

$$
e_{8}^{*}(\mathrm{z})=\frac{\mathrm{K}_{\mathrm{ni}} F_{i} \cdot U_{3} \mathrm{G} *(\mathrm{z})}{1+z^{-1} \cdot \mathrm{K}_{\mathrm{ni}} F_{i} T G *(z, 1)},
$$

где $\mathrm{G}^{*}(\mathrm{z}, 1)=\mathrm{z}_{\varepsilon=1}\left\{\frac{1}{T_{1} p}\right\}$.
Согласно с [7] модифицированное Z-преобразование передаточной функции $G(p)$ равно

$$
\mathrm{G} *(\mathrm{z}, 1)=\frac{1}{T_{1}} \cdot \frac{z}{z-1}
$$

С учетом Z-преобразования передаточной функции $G(p)$ характеристическое уравнение системы приобретает вид

$$
z-1+K_{\Pi i} F_{i} T=0
$$

Совместив корни характеристического уравнения (5) с началом Z-плоскости, получим условия процесса конечной длительности

$$
T_{1}=K_{I 1 \mathrm{i}} F_{i} T
$$

где $T$ - период дискретности ШИМ.

Для оценки динамических свойств системы рассмотрим ее реакцию на единое входное воздействие

$$
U_{3}(p)=L\{1(t)\}=\frac{1}{p} .
$$

Z-преобразование полинома числителя выражения (3) имеет вид

$$
z\left\{U_{3} G^{*}(z)\right\}=z\left\{\frac{1}{T_{1} p^{2}}\right\}=\frac{T}{T_{1}} \cdot \frac{z}{(z-1)^{2}} .
$$


С учетом формул (6) и (8) выражение для выходного сигнала (3) преобразуется к виду

$$
e_{6}^{*}(\mathrm{z})=\frac{\mathrm{z} \cdot \mathrm{K}_{\text {пі }} F_{i} \frac{T}{T_{1}}}{(z-1)\left(z-1+\mathrm{K}_{\text {пі }} F_{i} \frac{T}{T_{1}}\right)} .
$$

Выражение (9) описывает переходный процесс в системе при единичном входном воздействии.

Анализ выражений (6) и (9) указывает на то, что при строгом описании процессов в системе необходимо учитывать изменения на каждом интервале дискретности ШИМ статического $K_{n i}$ и динамического $F_{i}$ коэффициентов. Период повторяемости указанных коэффициентов определяется периодом дискретности выпрямителя.

Выражение для фактора пульсаций, записанное согласно [8] для $i$-го тактового интервала ШИМ, имеет вид

$$
\begin{array}{r}
F_{i}=\frac{1}{1+K_{n \mathrm{i}} \frac{T}{T_{1}}(1-\gamma)}, \quad(10) \quad \begin{array}{l}
\text { Лорана, получим } \\
\text { координаты систем } \\
\text { ШиМ }
\end{array} \\
e_{b}(n T)=z^{-1}+z^{-2}+z^{-3}+z^{-4}+\ldots+z^{-n} .
\end{array}
$$

где $\gamma-$ относительная длительность импульса ШИМ.

Из (10) следует, что изменение $K_{n i}$ вызывает соответствующее изменение $F_{i}$. Из этого следует, что изменение фактора пульсаций вызывает снижение чувствительности системы к изменениям ее параметров. Данное обстоятельство позволяет при анализе динамических процессов рассматривать в выражении (10) усредненные на интервале дискретности выпрямителе значения $K_{n}$.

При выборе из условия (6)

$$
K_{\Pi} \mathrm{F} \frac{\mathrm{T}}{\mathrm{T}_{1}}=1
$$

выражение для выходного сигнала (9) преобразуется к виду

$$
e_{B} *(\mathrm{z})=\frac{1}{z-1}
$$

Разложив (11) в степенной ряд Лорана, получим значения выходной координаты системы в тактовые моменты
Из (12) следует то, что переходный процесс в системе заканчивается за один тактовый интервал ШИМ, что соответствует порядку передаточной функции приведенной непрерывной части системы.

На рис. 3 приведена имитационная модель выпрямителя с ШИМ и системы автоматического регулирования, созданная в среде Matlab. Модель содержит трехфазную систему питающих выпрямитель с ШИМ напряжений $U_{\mathrm{A}}, U_{\mathrm{B}}$, $U_{\mathrm{C}}$. Выпрямитель представлен в виде блока Voltodobavka. Нагрузка выпрямителя выполнена на L1 и R1. Блок Step2 формирует скачек сигнала управления. Постоянная времени регулятора $G(\mathrm{p})$ выбрана в соответствии с условием процесса конечной длительности. На рис. 4 представлены осциллограммы переходного процесса и скачка сигнала управления. Верхняя осциллограмма характеризует изменение выходного сигнала регулятора в переходном режиме. Как следует из осциллограммы, переходный процесс в системе заканчивается за один тактовый интервал ШИМ, что подтверждает аналитический расчет. 


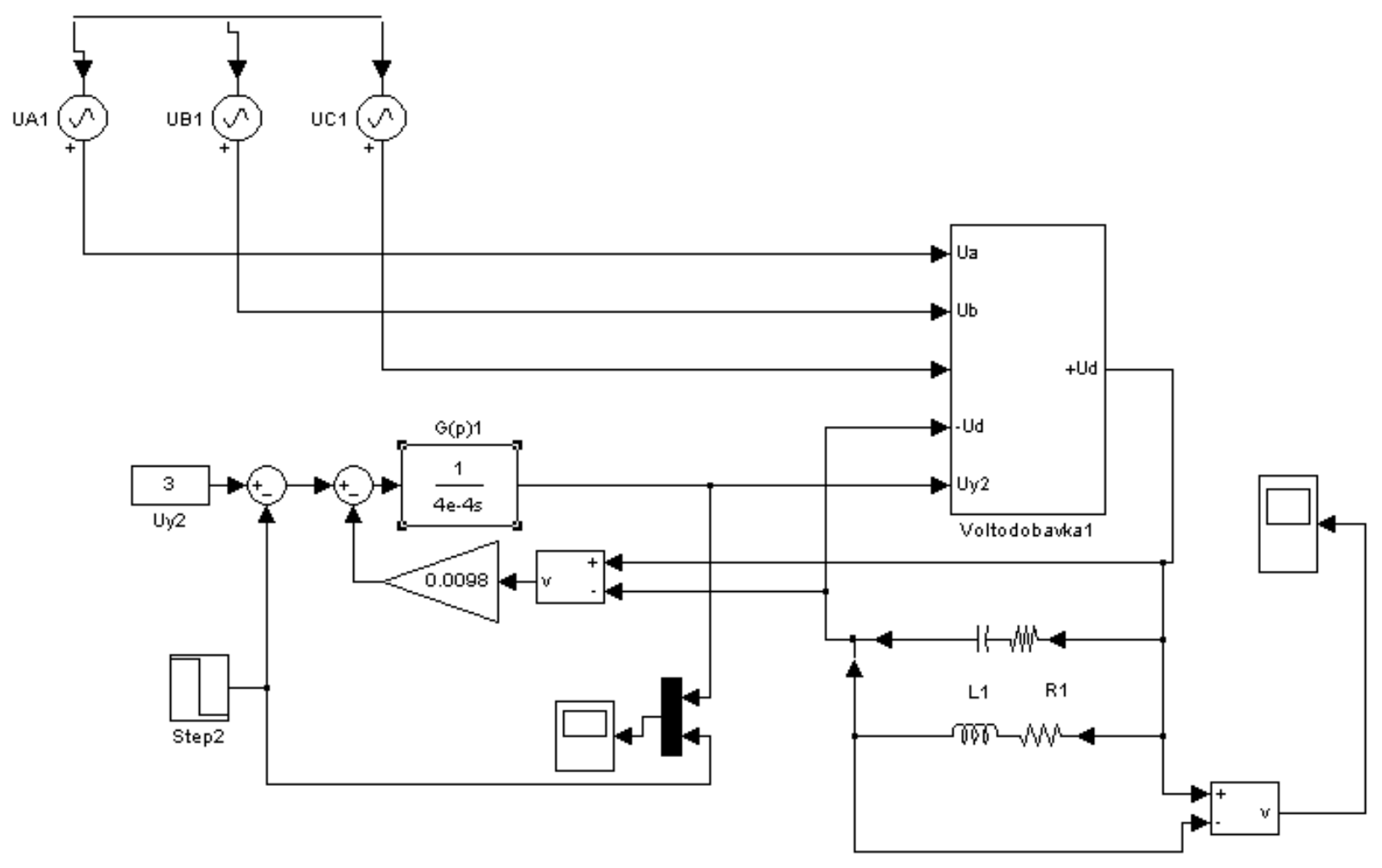

Рис. 3. Модель системы регулирования

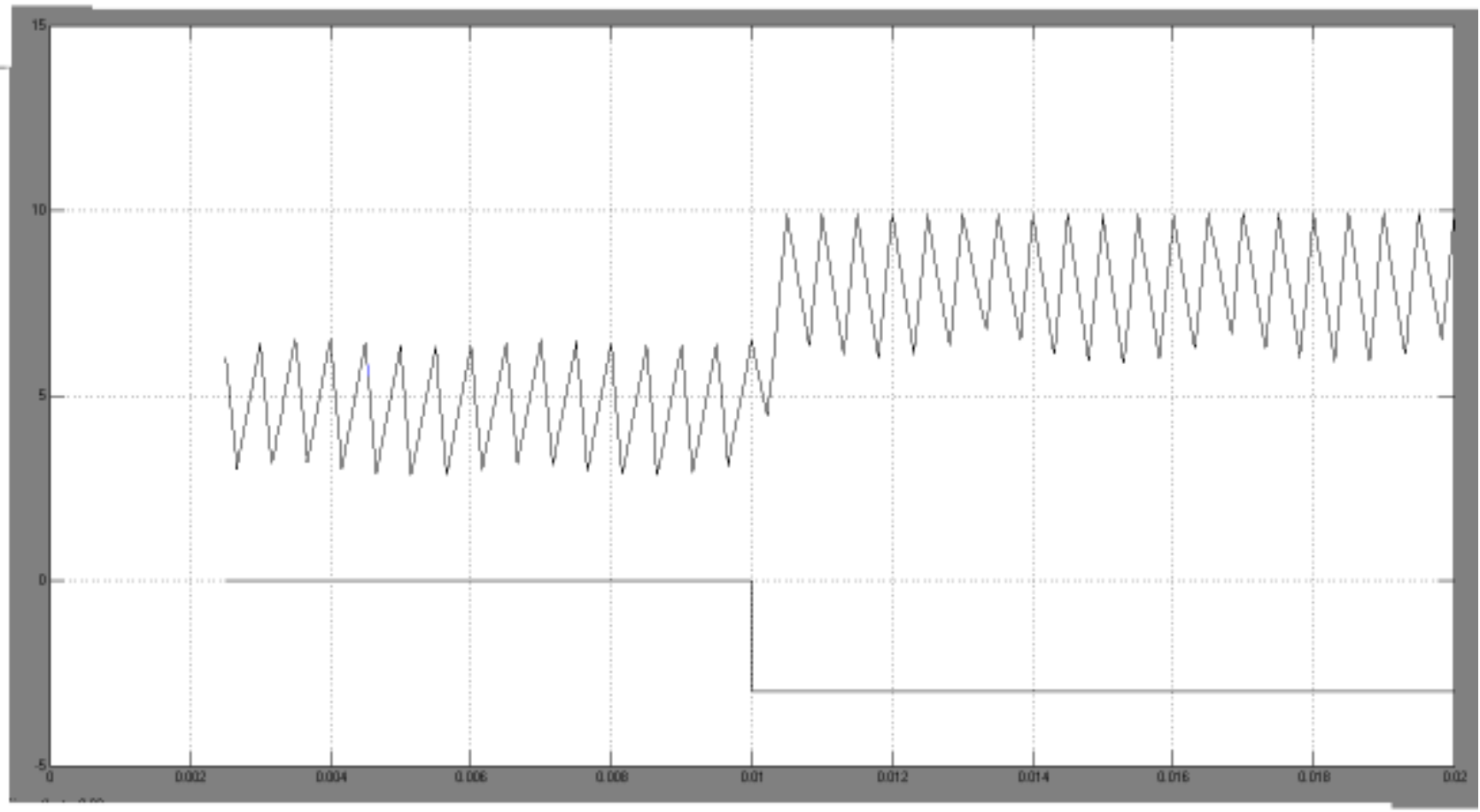

Рис. 4. Осциллограммы переходных процессов 
Выводы из исследований и перспективы, дальнейшее развитие в данном направлении. $\mathrm{B}$ результате выполненных исследований установлено, что применение усредненных значений статического коэффициента передачи и фактора пульсаций позволяет с достаточной точностью описывать динамические процессы в замкнутых структурах, содержащих выпрямитель с ШИМ.

\section{Список использованных источников}

1. Шипилло, В.П. Вентильный преобразователь как элемент системы автоматического регулирования [Текст] / В.П. Шипилло //Электричество. - 1967. - №11. - С.63-70.

2. Донской, Н.В. Управляемый выпрямитель в системах автоматического управления [Текст] / Н.В. Донской, А.Г. Иванов, В.М. Никитин, А.Д. Поздеев. - М.: Энергоатомиздат, 1984. $-352 \mathrm{c}$.

3. Чикотило, И.И. Исследование устойчивости и переходных режимов тиристорных широтно-импульсных преобразователей в быстродействующих замкнутых системах [Текст]: дис... канд. техн. наук: 05.09.12 / И.И. Чикотило. - Харьков, 1979. - 239 с.

4. Щербак, Я.В. Теоретические основы и методы регулирования субгармоник полупроводниковых преобразователей электроэнергии [Текст]: дис... д-ра техн. наук: 05.09.12 / Я.В. Щербак. - Харьков, 2001. - 408 с.

5. Щербак, Я.В. Широтно-импульсный преобразователь с широтно-импульсной модуляцией второго рода в условиях несимметрии [Текст] / Я.В. Щербак // Технічна електродинаміка. - К., 1999. - №1. - С.31-35.

6. Розанов, Ю.К. Основы силовой электроники [Текст] / Ю.К. Розанов. - М.: Энергоатомиздат, 1992. - 296 с.

7. Джури, Э. Импульсные системы автоматического регулирования [Текст] / Э. Джури. - М.: Физматиздат, 1963. - 455 с.

8. Шипилло В.П., Фактор пульсаций в системах регулирования с вентильными преобразователями [Текст] / В.П. Шипилло, Ю.С. Зинин //Электричество. - 1977. - №3. C. 86-89.

Рецензент д-р техн. наук, профессор Ю.И.Гусевский

Івакіна Катерина Яківна, аспірантка Харківського національного університету міського господарства.

Ivakyna Catherine Yakovlevna, postgraduate Kharkiv National University city-ray economy. 